anstieg. Als wichtiges Datum in der Geschichte der SVZ ist der 21. September 1939 zu nennen, denn damals schuf das Parlament die Voraussetzungen, um das Agenturnetz der SBB der SVZ einzuverleiben, was 1941 erfolgte. Mit dem gleichen Bundesbeschluß wandelte man letztere in eine öffentlich-rechtliche Körperschaft um, die den Namen «Schweizerische Zentrale für Verkehrsförderung» erhielt. 1955 wurde schließlich wieder die ursprüngliche Bezeichnung «Schweizerische Verkehrszentrale» eingeführt, die bis heute verwendet wird.

Laut Organisationsstatut organisiert und führt die SVZ die gesamtschweizerische Verkehrswerbung im Inund Ausland durch, wobei auch das Verständnis für die politische, kulturelle und wirtschaftliche Eigenart der Schweiz geweckt werden soll. Zu diesem Zweck arbeitet die Verkehrszentrale auf lokaler, regionaler, kantonaler, nationaler und internationaler Ebene eng mit den verschiedensten Fachstellen und Kommissionen zusammen.

Getragen wird die SVZ in erster Linie vom Bund, der rund zwei Drittel an das Budget in der Höhe von 28,5 Mio. Franken (1983) beisteuert. Andere namhafte Träger sind die SBB, die PTT, der Schweizer HotelierVerein, die Schweizerische Straßenverkehrswirtschaft sowie die Swissair. $\mathrm{Zu}$ den übrigen der insgesamt etwas mehr als 400 Mitgliedern zählen alle 26 Kantone, eine Reihe von Gemeinden und vor allem die im Tourismus oder in dessen Umfeld tätigen Organisationen.
Am Hauptsitz in Zürich sind rund 50 Personen mit journalistischen Arbeiten, der Planung und Durchführung von Ausstellungen und touristischen Werbereisen, der Herstellung einer vielfältigen Palette an Informationsbroschüren, allgemeinen Publikationen, Fotos, Plakaten, Presseartikeln usw. sowie mit administrativen Aufgaben beschäftigt. Jährlich werden um die 600 Tonnen an Imprimaten an die Werbefront im Ausland, das heißt an die auf alle fünf Kontinente verteilten SVZ-Agenturen verschickt. In den insgesamt 24 Vertretungen sind 200 weitere Mitarbeiter tätig, denen insbesondere Informationsaufgaben sowie die Kontaktpflege mit den Medien und touristischen Vereinigungen des Gastlandes obliegen. Überdies ist ein Teil der Agenturen mit dem Verkauf von Billetten der Transportanstalten des öffentlichen Verkehrs beauftragt.

Die SVZ versucht nicht nur, mit ihren Partnern zusammen einen hohen und überzeugenden Wirkungsgrad der Tourismuswerbung zu erreichen, sondern sie ist auch bestrebt, in unserer Wirtschaftspolitik eine aktive Rolle zu spielen. Schließlich engagiert sich die Schweizerische Verkehrszentrale mit anderen Institutionen ganz generell für die Präsenz unseres Landes im Ausland, etwa nach dem Motto: «Die Schweiz - ein schönes Stück Europa».

Schweizerische Verkehrszentrale (SVZ)

Bellariastr. 38, 8027 Zürich, Tel. 01/202 3737

\title{
Der Schweizerische Fremdenverkehrsverband (SFV)
}

Der Schweizerische Fremdenverkehrsverband (SFV) wurde 1932 in einer für den Tourismus schwierigen Zeit gegründet. Als tourismuspolitischer Dachorganisation wurde ihm die Vertretung der mit dem Fremdenverkehr zusammenhängenden Gesamtinteressen übertragen. Oberste Zielsetzung des SFV ist somit, zu einer langfristigen Verbesserung des Angebotes des Reise- und Ferienlandes Schweiz und damit zur Förderung des drittwichtigsten Wirtschaftszweiges und des zweitwichtigsten Arbeitgebers beizutragen. Im Gegensatz zur Schweizerischen Verkehrszentrale oder zu den SBB verzichtet der SFV aber auf jegliche Publizität oder Tätigkeit, die der eigentlichen Reisewerbung und der Fremdenverkehrspropaganda dient. Der SFV ist als privatrechtlicher Verein organisiert, dem heute über 630 Mitglieder angehören. Er hat seinen Sitz in Bern und beschäftigt dort ein Team von zehn Mitarbeitern (Ökonomen, Juristen, PR-Fachleute und kaufmännisch ausgebildetes Personal).

Eine Hauptaufgabe des SFV besteht in der repräsentativen Vertretung der schweizerischen Fremdenverkehrswirtschaft. Dies setzt die Verbandszugehörigkeit möglichst vieler Tourismusträger voraus. Mit der Einsitznahme sämtlicher Kantone, von 75 Fremdenverkehrsgemeinden, über 200 nationalen, regionalen und lokalen touristischen Verbänden, Institutionen und Vereinen, 176 Bergbahnen, Hotels und Restaurants, 39 Banken und Versicherungen, 31 Architekturund Planungsbüros sowie von 78 sonstigen dem Fremdenverkehr nahestehenden Einzelunternehmungen in den Organen des SFV dürfte die Forderung nach einer umfassenden Mitgliedschaft erfüllt sein. 
Für eine wirksame Tätigkeit des SFV im Bereich der Fremdenverkehrspolitik unerläßlich ist sodann die Ausrichtung der Fremdenverkehrswirtschaft auf ein gemeinsames Ziel.

Die Interessenwahrung und Mitsprache der Tourismusträger bei allen fremdenverkehrspolitischen Entscheidungen ist eine weitere wichtige Aufgabe des SFV. Im Verlaufe des Gesetzgebungsverfahrens spielen - neben den Gesprächen in den parlamentarischen Vorzimmern sowie der Einsitznahme von Verbandsexponenten in der Legislative - vor allem die Stellungnahmen im Rahmen der Vernehmlassungsverfahren eine zentrale Rolle. In aufwendiger Arbeit werden in dieser Phase Grundsatzüberlegungen zu kommenden Gesetzesvorlagen angestellt und vorausschauend die sich für den Tourismus ergebenden Konsequenzen beurteilt und öffentlich dargelegt. Dabei werden die sich stellenden Fragen gemeinsam mit den wichtigsten im Tourismus tätigen Institutionen angegangen, insbesondere mit der Schweizerischen Verkehrszentrale, dem Schweizer Hotelier-Verein, dem Schweizer Wirteverband sowie dem Verband Schweizerischer Kur- und Verkehrsdirektoren. Unter der Federführung des SFV wurde so in den letzten fünf Jahren zu 20 Bundesgesetzen oder Verordnungen eine Vernehmlassung eingereicht, u. a. zu den Richtlinien betreffend Eingriffe in die Landschaft im Interesse des Skisportes, zur Verordnung über die Konzessionierung von Luftseilbahnen, zum Umweltschutzgesetz, zur Verordnung über die Benützung des eidg. Kartenwerkes und der Pläne der Grundbuchvermessung, zur Gesamtverkehrs- und zur Gesamtenergiekonzeption, zur Lex Furgler sowie auch zum Fußund Wanderweggesetz. Die wichtige Funktion der Mitsprache erfüllt der SFV sodann auch auf interna- tionaler Ebene, nämlich als Mitglied der Schweizer Delegation im OECD-Tourismuskomitee.

Als dritter wesentlicher Aufgabenbereich obliegt dem SFV die Informations- und Beratungstätigkeit. $\mathrm{Zu}$ diesem Zweck ist dem Verband eine Dokumentationsund Beratungsstelle angegliedert, die sich vor allem mit der Erarbeitung von Grundlagen zu einer langfristigen Verbesserung des touristischen Angebotes der Schweiz befaßt. So werden im In- und Ausland umfassende Informationen und Erfahrungen gesammelt und in einschlägigen Berichten ausgewertet. In jüngster Vergangenheit wurden Themen wie «Sportanlagen in Ferienorten", "Die Kurtaxe - eine taugliche Finanzquelle?» oder "Aparthotels - Entwicklung der Hotellerie auf neuen Wegen?» abgehandelt, Probleme bezüglich des Verkehrs in Naherholungsgebieten oder hinsichtlich des Campingtourismus untersucht sowie Leitbilder für verschiedene Fremdenverkehrsorte und -regionen erstellt. An die Kosten dieser gemeinwohlorientierten Tätigkeit leistet der Bund einen jährlichen Beitrag von 100000 Franken.

Nicht zuletzt möchte der SFV mit gezielter Öffentlichkeitsarbeit über die Bedeutung des Tourismus informieren und zu einem vermehrten Tourismusbewußtsein in der Bevölkerung beitragen. Zu diesem Zweck gibt er u.a. ein vierteljährlich erscheinendes Bulletin heraus. Mit seinen Informationen will der SFV indessen nicht ein rosarotes Bild der Fremdenverkehrswirtschaft malen, sondern er bemüht sich, Probleme und Lösungsmöglichkeiten stets offen darzulegen.

Schweizerischer Fremdenverkehrsverband (SFV)

Monbijoustr. 130, 3001 Bern, Tel. 031/45 8745

\section{Das Forschungsinstitut für Fremdenverkehr der Universität Bern}

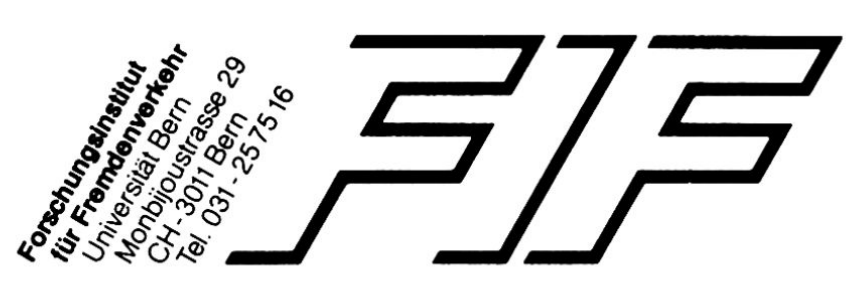

Mitten in den politischen und wirtschaftlichen Krisenjahren wurde 1941 an der Universität Bern das Forschungsinstitut für Fremdenverkehr (FIF) gegründet. Den damaligen Initianten darf ein hohes $\mathrm{Ma} B$ an Mut und Weitblick attestiert werden, war doch seinerzeit der Fremdenverkehr noch kaum ein hochschulfähiges Nebenfach. Die Leitung des Instituts war zu Beginn dem Inhaber des ordentlichen Lehrstuhls für Betriebswirtschaft (Prof. A. Walther) nebenamtlich übertragen. Aufgrund dieser Doppelfunktion konnte er nur zirka einēn Fünftel seiner Tätigkeit zugunsten 